\section{Effects of induced muscle tension upon the visual evoked potential and motor potential*}

\author{
J. L. ANDREASSI, M. S. MAYZNER, D. R. BEYDA, and S. DAVIDOVICS \\ New York University, Bronx, New York 10453
}

Averaged evoked cortical potentials were recorded from over the visual and motor areas of four Ss in several experimental sessions which took place over a 4-week period. The visual evoked potential (VEP) and motor potential (MP) were recorded under each of four conditions: (1) light flash alone, (2) squeezing dynamometer alone, (3) light plus squeezing, and (4) resting. The major findings were that: (1) VEP is of greater magnitude with muscle tension than with light alone and (2) the MP was of greater magnitude under the squeezing plus light condition compared with squeezing alone. The enhancement effect in both types of potentials was attributed to the arousing influence of increased reticular activity.

There appears to be little in the literature concerning the effects of induced muscie tension (IMT) or other accessory stimulation upon the visual evoked potential (VEP) in humans. In what may be the only previous study of this nature, Eason, Aiken, White, \& Lichtenstein (1964) measured VEP to flashes of light while two Ss maintained a 25-1b force on a hand grip. The muscular work increased the amplitude of VEPs to light flashes. In one other S, VEPs were measured before and after he engaged in $40 \mathrm{sec}$ of physical exercise ("jumping jacks"). The VEPs increased in amplitude following exercise. The present experiment was designed to expand that of Eason et al (1964) by measuring motor potentials (MPs) in addition to VEPs during an IMT task, making use of several $S s$ in each experimental condition and by specifying evoked potential amplitude differences, if any, in terms of microvolts of activity.

\section{SUBJECTS}

The Ss were four male graduate students ranging from 22 to 28 years of age. None had visual defects other than myopia (corrected to 20/20).

APPARATUS AND PROCEDURE

At least two practice sessions were run with each $S$ and a number of evoked potential tracings were obtained prior to actual data collection. Ss were seated in an isolated laboratory room with two Es present and with ambient illumination of $40 \mathrm{fc}$ at eye level. The EEG was recorded by means of two silver-coated cup electrodes (Grass type E5) placed at 02 and C3 according to the "Ten-Twenty" International System (Jasper, 1958). The

*This research was supported by the Physiological Psychology Program, Office of Naval Research, under ONR Contract No. N00014-67-A-0467-0009 and ONR Contract Authority No. NR 140-252 to the first tito authors. electrode leads from 02 and $\mathrm{C} 3$ were referred to a Grass silver clip electrode on the left earlobe. $S$ was grounded by means of an electrode-strap combination attached to the inner surface of his left wrist. Eye movements were recorded by placing another cup electrode over the left eyebrow such that it was aligned with the center of the pupillary aperture when $S$ looked straight anead. This was done to monitor possible eye movement (EOG) artifact in the EEG record.

EEG and EOG potentials were fed into a Mnemotron computer of average transients (CAT/1000) to produce averaged VEP, MP, and EOG. Samples of EEG and EOG activity of $500-\mathrm{msec}$ duration following each stimulus were summated and, upon termination of a given trial, traced out with an Hewlett-Packard 7004A X-Y Plotter. These physiological data were obtained under the following experimental conditions: (1) Resting-S was told to "close your eyes and maintain a blank mind" while 120 samples of resting activity were taken. (2) Light only-S was told to "concentrate on the flashing light and try not to blink." (Intensity No. 1 of a Grass PS 2 photo stimulator was presented at a rate of $1 \mathrm{~Hz}$ for all VEP conditions.) (3) Squeezing only -S squeezed a Stoeliting dynamometer handle at $1 / 8$ of his maximum grip (the average of three "maximum effort" squeezes was taken for each of the Ss, who were all right-handed). $S$ squeezed the handle at a rate of one squeeze per second and was assisted in his timing by a metronome that clicked in the background at this rate. $\mathrm{S}$ was instructed to "close your eyes and concentrate on squeezing the handle. You will hear clicks in the background, try to squeeze the handle just after each click occurs." The sweeps of the CAT were triggered by a circuit completed at the instant $S$ squeezed the dynamometer at the appropriate level. A "stop" was provided to insure that $S$ did not squeeze beyond the desired level. (4) Squeezing and light-S was told to "concentrate on both the flashing light and squeezing the handle just after each click occurs, and try not to blink." The photo stimulator was triggered when $S$ squeezed the dynamometer at the required level. The CAT was triggered simuitaneousily with the photostimulator.

One hundred and twenty samples were taken under each condition. The conditions were counterbalanced and Ss rested after each set of 120 stimuli. The entire session typically lasted from 2 to $2 \frac{1}{2} \mathrm{~h}$, with three tracings for each condition being obtained. Experimental data were collected over a 4-week period. Two of the Ss had 8 traces per condition, one had 10, and one had 12 traces per condition. The difference in number of tracings was due to scheduling problems. The Ss reported at the same time of day each week for the experimental sessions.

RESULTS AND DISCUSSION

The mean amplitudes and latencies for each of the major components (N1, Pl, $\mathrm{N} 2$, and $\mathrm{P} 2$ ) of the evoked response were computed for each condition for each of the four Ss. The ampitude of the NI component was measured from baseline to the trough of the first negative deflection which occurred after $50 \mathrm{msec}$. The Pl component was measured as the vertical distance from N1 (trough) to the peak of the first positive component, while N2 was taken as the vertical distance between the peak of P1 to the trough of the second major deflection in the wave form, and P2 was measured as the vertical distance between N2 and the peak of the second positive component. Latencies were measured to the midpoints of each positive and negative peak. If the "peak" was flat,

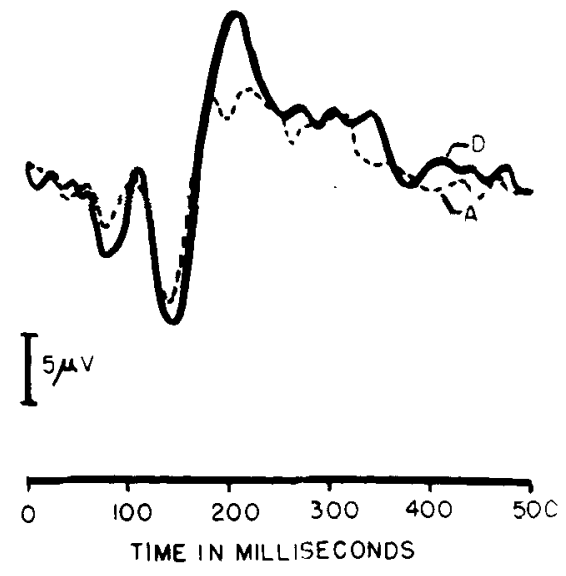

Fig. 1. VEP recorded from one S (DB) for Conditions $D$ (light and squeezing) and A (light alone). Each tracing based on responses to 120 light flashes at $1 \mathrm{~Hz}$. Negativity is downward. 


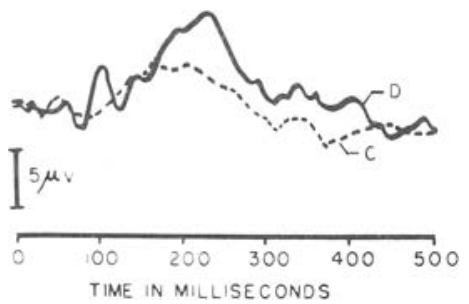

Fig. 2. MP recorded from one S (DB) for Conditions $D$ (light and squeezing) and $C$ (squeezing alone). Each tracing based on 120 samplings at rate of one sample per second. Negativity is downward.

the midpoint of the plateau was taken for the latency measurement.

The mean amplitudes for the various conditions for both VEP and MP are shown in Table 1. The data show that for both VEP and MP, Condition D (squeezing and light) yielded the largest evoked potentials (except P1 for VEP). This finding is compatible with the results reported by Eason et al (1964) indicating an enhancement of the VEP with IMT. Table 1 also shows that the MP is affected by responses in the visual area to a greater extent than the visual responses are influenced by motor activity. Evidence for this statement can be seen in Table 1, i.e., the VEP.P2 component is increased from 13.57 microvolts (Condition A) to 15.79 microvolts (Condition D), while the MP-P2 component increased from 5.44 microvolts (Condition C) to 9.50 microvolts (Condition D).

A comparison of Conditions A (light alone) and $C$ (squeezing alone) indicates that VEP is of greater magnitude than MP
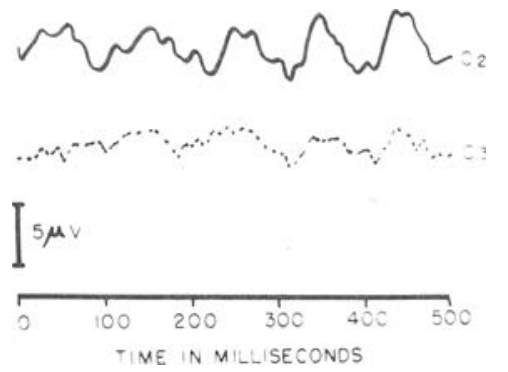

Fig. 3. Average resting brain activity recorded from 02 (visual area) and $\mathrm{C} 3$ (motor area), for one S (DB), 120 resting samples taken at rate of one sample per second. Negativity is downward.

for each waveform component. In addition, Condition $A$ produced a definable MP from $\mathrm{C} 3$, while Condition $C$ did not produce a definable VEP response from the visual area 02 . The average resting activity from the visual area was 4 microvolts compared with an average of 3 microvolts from the motor area, perhaps because relatively higher amplitude alpha activity is dominant during resting in the visual area, while the motor area shows a combination of alpha and beta waves.

Figures 1, 2, and 3 show copies of typical raw tracings for one of the $\mathrm{Ss}$ during a single experimental session. Figure 1 shows the increased amplitude of the VEP with IMT for Conditions A and D. Figure 2 shows how the MP was influenced by the visual stimulus. Samples of average resting responses for the visual and motor areas are depicted in Fig. 3. The average resting EEG for the visual area shows a stronger alpha response (i.e., five cycles in

Table 1

Mean Amplitude (in microvolts) for VEP and MP Components Under Conditions of This Experiment

\begin{tabular}{|c|c|c|c|c|c|}
\hline & & N1 & $\mathbf{P l}$ & $\mathrm{N} 2$ & $\mathrm{P} 2$ \\
\hline & & \multicolumn{4}{|c|}{ VEP Component (from O2) } \\
\hline A & (Light Alone) & 3.35 & 7.39 & 11.05 & 13.57 \\
\hline C & (Squeezing Alone) & \multicolumn{4}{|c|}{ (Average Activity Level of $5 \mu \mathrm{v}$ ) } \\
\hline $\mathrm{D}$ & (Squeezing and Light) & 3.68 & 7.28 & 11.70 & 15.79 \\
\hline B & (Rest) & \multicolumn{4}{|c|}{ (Average Activity Level of $4 \mu \mathrm{v}$ ) } \\
\hline & & \multicolumn{4}{|c|}{ MP Component (from C3) } \\
\hline A & (Light Alone) & 2.99 & 3.98 & 5.55 & 8.84 \\
\hline $\mathrm{C}$ & (Squeezing Alone) & 2.90 & 3.86 & 3.43 & 5.44 \\
\hline $\mathrm{D}$ & (Squeezing and Light) & 3.12 & 5.06 & 5.49 & 9.50 \\
\hline B & (Rest) & \multicolumn{4}{|c|}{ (Average Activity Level of $3 \mu \mathrm{v}$ ) } \\
\hline
\end{tabular}

Table 2

Mean Latency (in milliseconds) for VEP and MP Components Under Conditions of This Experiment

\begin{tabular}{llcccc}
\hline & & N1 & P1 & N2 & P2 \\
\hline A & (Light Alone) & \multicolumn{5}{c}{ VEP Component (from O2) } \\
C & (Squeezing Alone) & 70.64 & 105.10 & 143.57 & 204.49 \\
D & (Squeezing and Light) & -71.46 & 104.37 & 141.15 & 207.05 \\
& & \multicolumn{5}{c}{ MP Component (from C3) } \\
A & (Light Alone) & 74.68 & 100.89 & 134.58 & 216.28 \\
C & (Squeezing Alone) & 72.36 & 117.23 & 146.81 & 200.11 \\
D & (Squeezing and Light) & 70.59 & 108.58 & 128.58 & 197.52 \\
\hline
\end{tabular}

$500 \mathrm{msec}$ ) than does the motor area, as would be expected.

Table 2 shows the mean latency data (in milliseconds) for both the VEP and MP components under the various conditions. Examination of Table 2 reveals no latency difference between Conditions $A$ and $D$. Condition $\mathrm{C}$, which is a measure of activity in the visual area with the eyes closed (squeezing alone), showed no definable waveform and such is indicated by the blank spaces for the various components in Table 2. With respect to MP latency. the data in Table 2 indicate slightly shorter latencies under Condition $D$ than for Conditions $A$ and $C$.

The two most important findings of the present study were that: (1) VEP was greater in magnitude with IMT than with light alone, expecially for P2 (15\% increase), and (2) the MP was of greater magnitude under the squeezing and light condition compared with the squeezing alone condition (an increase in P2 of $75 \%$ ). There are references in the literature that lead us to hypothesize that the reason for the enhancement effects in both types of potentials is the arousing influence of the ascending reticular activating system (ARAS). For example, Lindsley (1958) reported that mild electrical stimulation of the reticular formation led to changes in the VEP recorded directly from the visual cortex of cats. Prior to stimulation the cat gave only one evoked response to two flashes of light presented $50 \mathrm{msec}$ apart, while for $10-12 \mathrm{sec}$ after reticular stimulation two evoked responses occurred to two stimuli $50 \mathrm{msec}$ apart. Chang (1959) indicates that microelectrode recordings from thalamic and cortical neurons show that as a stimulus increases in strength, the number of recorded spike potentials increases. If one considers that sensory stimuli can interact at the level of the reticular formation (Chang, 1959; Samuels, 1959), then it might be possible that impulses arising from proprioceptive stimuli are added to those produced by visual stimuli to increase the magnitude of each response in the appropriate cortical area.

There are behavioral studies of humans which indicate that cortical evoked potential amplitude can be related to performance. For example, Haider, Spong, \& Lindsley (1964) found that drops in VEP magnitude were related to decrements in visual vigilance performance. A similar result was reported by Wilkinson, Morlock, \& Williams (1966) for auditory evoked potentials recorded during an auditory vigilance task. Groves \& Eason (1969) concluded from experimental results that the attentional aspects of having Ss respond within a specified period to avoid 
shock was important in increasing the amplitude of the VEP. Simply responding or receiving an occasional shock was not sufficient to enhance VEP. In addition, a study by Shagass, Haseth, Callaway, \& Jones (1968) provides some evidence that higher amplitudes of evoked potentials were related to superior performance in a variety of perceptual tasks, including letter perception, lifted weights, and two-flash threshold.

The finding in our present experiment that VEP (light alone) is of greater magnitude than MP (squeezing alone) can lead to no firm statement regarding the Basis for this difference. The only conclusion justifiable is that the intensity of the visual stimulus used provided more excitation to the visual system than the particular level of IMT chosen provided to the motor area.

The finding that the MP was affected to a greater extent by visual stimuli than the VEP was affected by motor activity was unexpected. Two possible explanations are: (1) The intensity of the visual stimulus used to obtain VEPs was relatively greater than the intensity of the muscle activity required for obtaining the MP and, therefore, had more influence on evoked potentials recorded from both areas; and (2) since sensory neurons are located in the fourth layer of the visual cortex (Area 17 see Morgan, 1965, p. 49) while motor neurons are in the fifth layer of the motor cortex (Area 4, see Morgan, 1965, p. 49), greater attenuation of motor impulses may occur because they must pass upward through four layers to reach the cortical surface, while visual impulses must pass through only three other cortical layers to reach the surface. The first of these hypotheses is preferred because it is the simpler of the two and is related to the finding that the VEP to light alone was greater in magnitude than the MP to squeezing alone in the present experiment. However, the second hypothesis points to a possible contributing factor in studies of this type and could perhaps be tested out in future experiments with lower animals.

\section{REFERENCES}

CHANG, H. T. The evoked potentials. In $\mathrm{H}$. W. Magoun (Ed., Neurophysiology section), Handbook of physiology, Vol. I. Baltimore: Williams \& Wilkins, 1959.

EASON, R. G., AIKEN, L. R., WHITE, C. T., \& LICHTENSTEIN, M. Activation and behavior: II. Visually evoked cortical potentials in man as indicants of activation level. Perceptual \& Motor Skills, 1964, 19, 875-895.

GROVES, P. M., \& EASON, R. G. Effects of attentiation and activation on the visual evoked cortical potential and reaction time. Psychophysiology, 1969, 5, 394-398.

HAIDER, M., SPONG, P., \& LINDSLEY, D. B. Attention, vigilance, and cortical evoked-potentials in humans. Science, 1964, 145, 180-182.

JASPER, H. H. Report of the committee on methods of clinical examination in e le c troe n c e phalog a phy. Electroencephalography \& Clinical Neurophysiology, 1958, 10,370-375.

LINUSLEY, $\boldsymbol{U}$. B. The reticular system and perceptual discrimination. In H. H. Jasper et al (Eds.), Reticular formation of the brain. Boston: Little, Brown, 1958. Pp. 513-534.

MORGAN, C. T. Physiological psychology. 3rd ed. New York: McGraw-Hill, 1965.

SAMUELS, I. Reticular mechanisms and behavior. Psychological Bulletin, 1959, 56, 1-25.

SHAGASS, C., HASETH, K., CALLAWAY, E., \& JONES, R. EEG-evoked response relationships and perceptual performance. Life Sciences, 1968, 7, 1083-1091.

WILKINSON, R. T., MORLOCK, H. C., \& WILLIAMS, H. L. Evoked cortical response during vigilance. Psychonomic Science, 1966, 4, 221-222.

\title{
Effects of reducing the readability of the words in the Stroop Color-Word Test
}

\author{
WILLIAM E. GUMENIK and ROBERT GLASS* \\ The University of Toledo, Toledo, Ohio 43606
}

Decreasing the readability of words, by partially obscuring them with a mask, increased the susceptibility of reading and decreased the susceptibility of color naming to interference in the Stroop color-word conflict situation. The conflict interfered with both reading and color-naming performance, with and without the mask, but the degree of interference was inversely related to the relative strength of the response.

In the Stroop Color-Word Test, color words are printed in noncongruent colors, i.e., the word blue printed in red. It takes longer to name the colors in which these words are presented than to name the colors in which abstract forms or noncolor words are printed. The commonest explanation of this phenomenon is that the competitive response of reading the color words is stronger than and interferes with color naming. Findings that color words are read faster than colors are named in nonconflict situations and that the reading/color-naming conflict interferes more with color naming than with word reading support this explanation (Jensen \& Rohwer, 1966; Stroop, 1935).

It would be expected, from this explanation, that weakening the reading response should reduce interfering effects of reading on color naming and increase the susceptibility of the reading response to interference from color naming in the conflict situation. It has been reported that some Ss attempt to maximize their color-naming performance in this conflict situation by squinting or deaccommodating (Jensen \& Rohwer, 1966). In the present experiment, the effects of partially masking the words, and therefore rendering them more difficult to read, on the susceptibility, of color naming and word reading, to interference in the conflict situation were investigated.

\section{SUBJECTS}

The Ss were 60 University of Toledo men and women student volunteers who were assigned randomly to four equal size groups of 15 Ss each.

\section{MATERIALS}

There were three $22 \times 18$ in. charts. Each chart consisted of 10 rows of eight words or nonsense syllables printed in 1/2-in.high gothic letters against a white background. The rows were separated by $11 / 2$-in. spaces. The rows on the word chart consisted of the words "red," "gold," "blue," and "green" printed in black ink and presented in block-randomized order, each word appearing twice in each row. The rows on the color chart consisted of the nonsense syllable OANS, printed in red, gold, blue, and green "Plas-Stick" tape, the color of the nonsense syllables being presented in block-randomized order, with each color appearing twice in each row. The rows on the conflict chart consisted of the words of the word chart presented in the colors of the color chart. Both the order of the words and colors were block randomized, each word and color appearing twice in each row, with the restriction that the word and the color in which it was presented should never agree.

A $22 \times 18$ in. white opaque mask that could be placed over the charts served as an interference grid. The mask had $1 / 4$-in.-wide slits, $1 / 4$ in. apart, at a $45-d e g$ angle slant,

* Now at the University of Maryland, College Park, Maryland 20742. 